\title{
Prevalência de transtornos mentais comuns entre profissionais de saúde
}

\author{
Prevalence of common mental disorders among health professionals \\ Prevalencia de trastornos mentales comunes entre profesionales de salud
}

\author{
Ana Paula Alves'; Leila Aparecida Kauchakje Pedrosa ${ }^{\text {II }}$; Marli Aparecida Reis Coimbra ${ }^{\text {III }}$; \\ Mário Alfredo Silveira Miranzi ${ }^{i v}$; Vanderlei José Hass ${ }^{V}$
}

\begin{abstract}
RESUMO: Este estudo objetivou verificar a prevalência de transtornos mentais comuns (TMC) entre os profissionais de saúde de um hospital de universitário. Trata-se de um estudo observacional, transversal com abordagem quantitativa, realizado com 359 profissionais de saúde, no Hospital de Clínicas da Universidade Federal do Triângulo Mineiro, Minas Gerais. A coleta de dados ocorreu entre os meses de junho a agosto de 2013. Os participantes responderam um instrumento contendo variáveis sociodemográficas e profissionais e outro para verificar a prevalência de TMC o Self Reporting Questionnaire. Entre os profissionais de saúde detectou-se uma prevalência geral de 27,9\% para TMC. Os resultados obtidos neste estudo evidenciaram a presença de TMC, sendo necessário propor medidas para promover à saúde dos profissionais de saúde.

Palavras-Chave: Pessoal da saúde; SRQ-20; saúde do trabalhador; transtornos mentais.
\end{abstract}

ABSTRACT: This study aimed at assessing the prevalence of common mental disorders (CMD) among health professionals of a teaching hospital. It is a quantitative cross-sectional observational study conducted with 359 health professionals, at Hospital de Clínicas da Universidade Federal do Triângulo Mineiro, Minas Gerais, Brazil. Data collection occurred from June to August, 2013. Participants answered both an instrument with professional and social and demographic variables and another one for assessing the prevalence of CMD - the Self Reporting Questionnaire. Among those health professionals, an overall $27.9 \%$ prevalence for CMD was observed. Results identified CMD. It is therefore necessary to propose health promotion measures for health professionals. Keywords: Health personnel; SRQ-20; worker's health; mental disorders.

RESUMEN: Este estudio pretende verificar la prevalencia de trastornos mentales comunes (TMC) entre los profesionales de sanidad de un hospital universitario. Se trata de un estudio observacional, transversal con abordaje cuantitativo, realizado con 359 profesionales de sanidad, en el Hospital de Clínicas de la Universidade Federal de Triangulo Mineiro, Minas Gerais. La recolecta de datos se produjo entre los meses de julio y agosto de 2013. Los partícipes respondieron un instrumento que describe variables sociodemográficas y profesionales y otro para verificar la prevalencia del TMC o Self Reporting Questionnaire. Entre los profesionales de salud se detectó una prevalencia general de 27,9\% para TMC. Los resultados obtenidos en este estudio destacaron la presencia de TMC, haciendo necesaria la propuesta de medidas de fomento de la salud de los profesionales sanitarios. Palabras Clave: Personal sanitario; SRQ-20; sanidad del trabajador; trastornos mentales.

\section{INTRODUÇÃO}

O exercício da atividade profissional permeia dimenções físicas, sociais e emocionais que são favoráveis ao equilíbrio mental, contentamento e desenvolvimento de capacidades, porém também pode ser causa de sofrimentos e esgotamento que por sua vez levam à alteração do estado de saúde do indivíduo ${ }^{1,2}$.

Os profissionais de saúde vivenciam inúmeras situações desgastantes na prática clínica, pois provém da frequente exposição a um ou mais elementos que favorecem o aparecimento de doenças ou de sofrimento, que são destacados por sinais e sintomas orgânicos e psíquicos, como os transtornos mentais, sendo que estes fatores afetam negativamente os resultados do trabalho e a qualidade da assistência ofertada pelos trabalhadores ${ }^{1,3,4}$.

Os transtornos mentais comuns (TMC) constituem os sintomas não- psicóticos caracterizados por queixas somáticas, insônia, mal estar gástrico, diminuição da concentração, irritabilidade, fadiga, sensação de inutilidade e dores de cabeça ${ }^{5}$. Os TMC são frequentemente encontrados em diversas populações, sua presença produz impactos em setores produtivos, bem como pode aumentar a demanda por serviços de saúde $e^{5-7}$.

'Enfermeira. Mestre em Atenção à Saúde da Universidade Federal do Triangulo Mineiro. Uberaba, Minas Gerais, Brasil. Email: apaula.alv@gmail.com IEnfermeira. Doutora em Saúde Coletiva. Docente da Universidade Federal do Triângulo Mineiro. Programa de Pós-graduação em Atenção à Saúde. Uberaba, Minas Gerais, Brasil. Email: leila.kauchakje@terra.com.br.

IIIEnfermeira. Mestre em Atenção à Saúde da Universidade Federal do Triângulo Mineiro. Uberaba, Minas Gerais, Brasil. Email: marli.apr.coimbra@gmail.com. IvOdontólogo. Doutor em Saúde Coletiva. Docente da Universidade Federal do Triângulo Mineiro. Uberaba, Minas Gerais, Brasil. Email: mmiranzi@mednet.com.br.

vFísico. Doutor em Física Aplicada à Medicina e Biologia. Docente da Universidade Federal do Triângulo Mineiro. Programa de Pós-graduação em Atenção à Saúde. Uberaba, Minas Gerais, Brasil. Email: vjhaas@uol.com.br. 
A literatura nacional acerca dos TMC entre profissionais de saúde ainda é escassa ${ }^{4}$. Diante do exposto, há a necessidade de uma exploração maior do tema, enfocando os diversos aspectos relacionados aos transtornos mentais. $\mathrm{O}$ presente estudo se propõe a verificar a prevalência de Transtornos Mentais Comuns entre os profissionais de saúde e analisar sua relação entre os fatores sociodemográficos e profissionais.

\section{REVISÃo DE Literatura}

Os transtornos mentais constituem $12 \%$ do total de doenças e incapacidades no mundo, sendo que um quarto das pessoas será afetada por um transtorno mental em alguma fase da vida, devido a natureza crônica que produz incapacitação são considerados um problema de saúde pública, pois causam estigmas e outros problemas ${ }^{8,9}$.

As doenças mentais apresentam difíceis diagnósticos precoces, podendo causar alterações psicoemocionais, orgânicas, sociais e culturais. Devido ao seu caráter subjetivo e ao estigma social que a patologia representa, este quadro resulta em deficiência de atendimento adequado a maioria da população em sofrimento mental ${ }^{8}$.

Os sintomas indicativos de TMC são mais frequentes entre pessoas mais velhas, sexo feminino, entre viúvos, além de estar relacionados aos eventos produtores de estresse, redução de apoio social, e com variáveis relativas às baixas condições de vida e de trabalho, menor renda e baixa escolaridade ${ }^{6,9,10}$.

As pesquisas epidemiológicas sobre saúde mental iniciaram-se em instituições hospitalares e serviços ambulatoriais, devido à necessidade de expandir os estudos sobre esse tema se estenderam às bases populacionais, sendo que os resultados demonstraram que cerca de $90 \%$ das manifestações psiquiátricas compõem-se de distúrbios não-psicóticos, incluindo depressão, ansiedade e sintomas como insônia, fadiga, estresse, dificuldade de memória e queixas somáticas ${ }^{11}$.

\section{Metodologia}

Trata-se de um estudo observacional, transversal com abordagem quantitativa, realizado no Hospital de Clínicas da Universidade Federal do Triângulo Mineiro. A população foi constituída por profissionais de saúde, foram selecionados empregando-se amostragem aleatória simples. O cálculo do tamanho amostral considerou uma prevalência de TMC de 32\%, uma precisão de $3 \%$ e um intervalo de confiança de $95 \%$, para uma população finita de 1.138 profissionais, chegando-se a amostra de 359 indivíduos, considerando uma perda de amostragem de $20 \%$, o número de tentativas de entrevistas máximo foi de 449. Foram adotados como critérios de inclusão no estudo ter: formação de enfermeiro, técnico em enfermagem, auxiliar de enfermagem, médico, psicólogo, fonodiólogo, assistente social, tera- peuta ocupacional e fisioterapeuta, e prestar assistência direta ao paciente. Foram excluídos os trabalhadores com menos de 30 dias de trabalho na instituição. A coleta de dados foi realizada de junho a agosto de 2013.

O presente trabalho fundamentou-se na Resolução no 466/12 do Conselho Nacional de Saúde do Brasil. O projeto de pesquisa foi submetido ao Comitê de Ética em Pesquisa com Seres Humanos sob o protocolo 2.505, sendo que os participantes assinaram o termo de consentimento livre e esclarecido.

A coleta de dados constou da aplicação de um formulário sociodemográfico e profissional e para analisar a prevalência de TMC foi utilizado o Self Reporting Questionnaire (SRQ-20), que avalia: queixas somáticas inespecíficas, irritabilidade, insônia, dores de cabeça, fadiga, esquecimento e dificuldade de concentração.

O SRQ-20 constitui a versão de 20 itens utilizados para rastreamento de transtornos mentais nãopsicóticos, desenvolvido pela Organização Mundial de Saúde (OMS) 8 e foi validado no Brasil ${ }^{12}$.

As respostas utilizadas no questionário são apenas sim ou não e cada resposta positiva corresponde ao valor 1 para compor o escore final por meio da soma total destes valores. Os escores obtidos são referentes à probabilidade de presença de transtorno não-psicótico, incluindo sintomas depressivos, ansiosos e queixas somáticas, variando de 0 que corresponde a nenhuma probabilidade e 20 que significa extrema probabilidade de apresentar $\mathrm{TMC}^{13}$.

Os sintomas foram agrupados em categorias, foi utilizada a classificação, que estabelece quatro subdivisões de sintomas que são o humor depressivo/ ansioso, sintomas somáticos, redução de energia vital e pensamentos depressivos ${ }^{14}$.

Os dados foram digitados em dupla entrada em planilha eletrônica (Programa Microsoft Office Excel ${ }^{\circledR}$ 2007 para Windows $\left.{ }^{\circledR}\right)$ e importados ao Statistic Package for Social Sciences (SPSS) versão 20.0.

Para análise das variáveis qualitativas foram utilizadas estatística descritiva e frequência absoluta e percentual. A análise bivariada incluiu medidas de associação em tabelas de contingência (qui quadrado, razão de prevalência e razão de chances de prevalência). A análise dos fatores de risco para a ocorrência ou não de TMC considerou um modelo de regressão logística binária, tendo como variáveis os preditores sociodemográficos e profissionais.

\section{Resultados e Discussão}

Entre os participantes da pesquisa, $18,4 \%$ eram médicos, $73,8 \%$ compunham a equipe de enfermagem e 7,8\% representavam os demais profissionais de saúde. Predominaram os profissionais do sexo feminino $77,2 \%$, que se declararam casados ou em união estável - 56,2\% - e possuíam filhos - 60,4\%. 
A média de idade dos participantes foi de 39,53 anos. A média de idade dos profissionais de saúde do hospital de ensino foi próximo, porém maior, ao encontrado em outro estudo ${ }^{15}$.

A predominância do sexo feminino, verificada neste estudo, tem consonância com outra pesquisa desenvolvida com profissionais de saúde ${ }^{15-17}$. A equipe de enfermagem representou a maior parte desse contingente, mostrando que profissão, ainda, permanece essencialmente feminina ${ }^{18-20}$. As mulheres desempenham papel fundamental na sociedade, pois além de estarem no mercado de trabalho, algumas vezes são as únicas provedoras da família, somando-se a este fato o trabalho doméstico que muitas vezes é realizado apenas por elas.

Do total de participantes, $74,4 \%$ possuíam 5 anos ou mais de atuação na instituição, 54,9\% trabalhavam no turno diurno, $23,1 \%$ realizavam suas atividades no turno noturno e $22 \%$ em mais de um turno, sendo este grupo representado pelos profissionais médicos - 71,2\%.

Entre os participantes da pesquisa, $67,1 \%$ declararam ter um vínculo empregatício, entretanto, entre os médicos, 72,7\% têm mais de um. A necessidade financeira e a busca por melhor remuneração motivam os profissionais a acumularem diversos empregos, apesar da rotina de trabalho desgastante ${ }^{21}$. Quanto à renda familiar, 50,1\% dos participantes da pesquisa declararam receber acima de 2 e até 6 salários mínimos, enquanto $14,2 \%$ tinham renda familiar de até 2 salários mínimos.

Entre os sintomas agrupados avaliados, através do SRQ-20, predominou a categoria de sintomas humor depressivo/ansioso, com destaque para a queixa sentir-se nervoso, tenso ou preocupado - $64,6 \%$, conforme mostra a Tabela 1. Esta categoria de sintomas foi também o mais prevalente em outros estudos, que utilizaram o mesmo instrumento de triagem ${ }^{22,23}$.

No grupo de sintomas somáticos, dormir mal representou $45,1 \%$ entre os sujeitos pesquisados. A questão referente ao sono também foi a queixa mais prevalente no grupo de sintomas somáticos analisados em outra pesquisa ${ }^{22}$, porém apresentou frequência superior a encontrada no presente estudo.

Entre os profissionais de saúde, do presente estudo, detectou-se uma prevalência de $27,9 \%$ de rastreamento positivo para TMC. A prevalência não foi tão elevada como em uma investigação realizada com trabalhadores da rede básica de saúde, em Botucatu, que demonstrou que $42,6 \%$ apresentaram indicativo de $\mathrm{TMC}^{24}$. Porém, a prevalência foi superior a encontrada em outra pesquisa realizada entre profissionais, na Bahia, em que prevalência foi de $16,0 \%{ }^{25}$. Era esperado que a prevalência de TMC fosse maior em profissionais que trabalham na área hospitalar do

TABELA 1: Prevalência de grupos de sintomas psíquicos em profissionais de saúde de um hospital de ensino, de acordo com o instrumento $S R Q=20$. Uberaba, 2013. $(N=359)$

\begin{tabular}{lcc}
\multicolumn{1}{c}{ Grupos de sintomas } & Sim & Não \\
& $\mathrm{f}(\%)$ & $\mathrm{f}(\%)$ \\
\hline Humor depressivo/ansioso & & \\
Q-4 Assusta-se com facilidade? & $83(23,1)$ & $276(76,9)$ \\
Q-6 Sente-se nervoso (a), tenso (a) ou preocupado (a)? & $232(64,6)$ & $127(35,4)$ \\
Q-9 Tem se sentido triste ultimamente? & $127(35,4)$ & $232(64,6)$ \\
Q-10 Tem chorado mais do que costume? & $61(17,0)$ & $298(83,0)$ \\
Sintomas somáticos & & \\
Q-1 Você tem dores de cabeça frequente? & $140(39,0)$ & $219(61,0)$ \\
Q-2 Tem falta de apetite? & $38(10,6)$ & $321(89,4)$ \\
Q-3 Dorme mal? & $162(45,1)$ & $197(54,9)$ \\
Q-5 Tem tremores nas mãos? & $50(13,9)$ & $309(86,1)$ \\
Q-7 Tem má digestão? & $118(32,9)$ & $241(67,1)$ \\
Q-19 Você se cansa com facilidade? & $117(32,6)$ & $242(67,4)$ \\
Decréscimo de energia vital & & \\
Q-8 Tem dificuldades de pensar com clareza? & $84(23,4)$ & $275(76,6)$ \\
Q-11 Encontra dificuldades para realizar com satisfação suas atividades diárias? & $108(30,1)$ & $251(69,9)$ \\
Q-12 Tem dificuldades para tomar decisões? & $78(21,7)$ & $281(78,3)$ \\
Q-13 Tem dificuldades no serviço (seu trabalho é penoso, lhe causa- sofrimento?) & $75(20,9)$ & $284(79,1)$ \\
Q-18 Sente-se cansado (a) o tempo todo? & $124(34,5)$ & $235(65,5)$ \\
Q-20 Têm sensações desagradáveis no estomago? & $124(34,5)$ & $235(65,5)$ \\
Pensamentos depressivos & & \\
Q-14 É incapaz de desempenhar um papel útil em sua vida? & $20(5,6)$ & $339(94,4)$ \\
Q-15 Tem perdido o interesse pelas coisas? & $101(28,1)$ & $258(71,9)$ \\
Q-16 Você se sente uma pessoa inútil, sem préstimo? & $19(5,3)$ & $340(94,7)$ \\
Q-17 Tem tido ideia de acabar com a vida? & $10(2,8)$ & $349(97,2)$ \\
\hline
\end{tabular}


que naqueles da atenção básica devido às diferentes complexidades, apesar de os estudos mostrarem resultados contraditórios.

A prevalência de TMC, encontrada neste estudo, foi superior à identificada entre trabalhadores, na Etiópia, que mostrou que neste país a presença para TMC atingiu 17,7\% da população geral, representando $25,9 \%$ de mulheres e $12,4 \%$ em homens ${ }^{26}$.

Quanto aos achados da análise bivariada do TMC, segundo as variáveis sociodemográficas entre dos profissionais de saúde, observou-se que a prevalência de TMC no sexo feminino foi de $32,5 \%$, enquanto que no sexo masculino atingiu $12,2 \%$; a razão de prevalência (RP) alcançou 2,66 ( IC 95\% 1,45-4,87), conforme a Tabela 2.
$\mathrm{Na}$ análise bivariada, a associação entre TMC e renda familiar foi estatisticamente significativa $(p=0,001)$, os profissionais que têm renda entre 1 e 2 salários mínimos apresentaram duas vezes mais TMC que aqueles com renda superior a 6 salários mínimos (RP: 2,39; IC 95\% 1,45-3,95), conforme expõe a Tabela 2. A prevalência para menor renda foi de 41,2\%, enquanto aqueles com ganhos acima de 2 e até 6 salários mínimos alcançaram $31,7 \%$ e acima de 6 salários foi de 17,2\% de rastreamento positivo para TMC.

Os resultados da análise bivariada do TMC, segundo as variáveis profissionais, estão especificados na Tabela 3 .

TABELA 2: Prevalência de transtornos mentais comuns entre profissionais de saúde em um hospital de ensino, segundo variáveis sociodemográficas. Uberaba, 2013. $(\mathrm{N}=359)$

\begin{tabular}{|c|c|c|c|c|c|c|c|}
\hline \multirow{3}{*}{ Variáveis } & \multicolumn{4}{|c|}{ TMC } & \multirow{3}{*}{ RP } & \multirow{3}{*}{ RCP (IC 95\%) } & \multirow{3}{*}{$P$} \\
\hline & \multicolumn{2}{|c|}{ Sim } & \multicolumn{2}{|c|}{ Não } & & & \\
\hline & $f$ & $\%$ & $f$ & $\%$ & & & \\
\hline \multicolumn{8}{|l|}{ Sexo } \\
\hline Feminino & 90 & 32,5 & 187 & 67,5 & $2,66(1,45-4,87)$ & $3,46(1,70-7,00)$ & $0,001^{(*)}$ \\
\hline Masculino & 10 & 12,2 & 72 & 87,8 & & & \\
\hline \multicolumn{8}{|l|}{ Idade } \\
\hline$\leq 39$ anos & 66 & 34,2 & 127 & 65,8 & $1,67(1,16-2,38)$ & $2,01(1,24-3,26)$ & $0,003^{(*)}$ \\
\hline$\geq 40$ anos & 34 & 20,5 & 132 & 79,5 & & & \\
\hline \multicolumn{8}{|l|}{ Estado conjugal } \\
\hline Sem companheiro & 48 & 30,6 & 109 & 69,4 & $1,18(0,85-1,65)$ & $1,27(0,79-2,01)$ & 0,186 \\
\hline Com companheiro & 52 & 25,7 & 150 & 74,3 & & & \\
\hline \multicolumn{8}{|l|}{ Número de filhos } \\
\hline Nenhum & 41 & 28,9 & 101 & 71,1 & $1,06(0,75-1,48)$ & $1,08(0,67-1,74)$ & 0,409 \\
\hline 1 ou mais & 59 & 27,2 & 158 & 72,8 & & & \\
\hline \multicolumn{8}{|l|}{ Renda familiar } \\
\hline 1 até 2 salários & 21 & 41,2 & 30 & 58,8 & $2,39(1,45-3,95)$ & $3,37(1,63-6,94)$ & $0,001^{(*)}$ \\
\hline Acima de 2 até 6 salários & 57 & 31,7 & 123 & 68,3 & $1,84(1,19-2,85)$ & $2,23(1,28-3,89)$ & \\
\hline Acima de 6 salários & 22 & 17,2 & 106 & 82,8 & & & \\
\hline
\end{tabular}

TABELA 3: Prevalência de transtornos mentais comuns entre profissionais de saúde em um hospital de ensino, segundo variáveis profissionais. Uberaba, 2013. $(\mathrm{N}=359)$

\begin{tabular}{|c|c|c|c|c|c|c|c|}
\hline \multirow{3}{*}{ Variáveis } & \multicolumn{4}{|c|}{ TMC } & \multirow{3}{*}{ RP } & \multirow{3}{*}{ RCP (IC 95\%) } & \multirow{3}{*}{$\mathbf{P}$} \\
\hline & \multicolumn{2}{|c|}{ Sim } & \multicolumn{2}{|c|}{ Não } & & & \\
\hline & $f$ & $\%$ & $f$ & $\%$ & & & \\
\hline \multicolumn{8}{|l|}{ Categoria profissional } \\
\hline Equipe de enfermagem & 89 & 33,6 & 176 & 66,4 & $3,69(1,69-8,07)$ & $5,05(2,10-12,00)$ & 0,090 \\
\hline Outros profissionais & 5 & 17,9 & 23 & 82,1 & $1,88(0,83-4,23)$ & $2,32(0,85-6,32)$ & 0,065 \\
\hline Médicos & 6 & 9,1 & 60 & 90,6 & & & \\
\hline \multicolumn{8}{|l|}{ Setor que trabalha } \\
\hline UTIs & 28 & 30,8 & 63 & 69,2 & $1,19(0,81-1,74)$ & $1,27(0,74-2,19)$ & 0,344 \\
\hline Emergências & 18 & 30,5 & 41 & 69,5 & $1,18(0,75-1,84)$ & $1,26(0,66-2,37)$ & \\
\hline $\begin{array}{l}\text { Enfermarias, bloco cirúrgico, } \\
\text { ambulatórios }\end{array}$ & 54 & 25,8 & 155 & 74,2 & & & \\
\hline \multicolumn{8}{|l|}{ Número de horas trabalhadas } \\
\hline $20-44$ horas & 69 & 29,5 & 165 & 70,5 & $1,182(0,82-1,71)$ & $1,26(0,77-2,07)$ & 0,207 \\
\hline \multicolumn{8}{|l|}{ Turno de trabalho } \\
\hline Noturno & 29 & 34,9 & 54 & 65,1 & $3,42(1,41-8,26)$ & $4,72(1,68-13,22)$ & $0,003^{(*)}$ \\
\hline Diurno & 71 & 25,7 & 205 & 74,3 & $2,84(1,21-6,70)$ & $3,60(1,37-9,50)$ & \\
\hline Mais de um turno & 5 & 10,2 & 44 & 89,8 & & & \\
\hline \multicolumn{8}{|l|}{ Formação acadêmica } \\
\hline Ensino médio & 49 & 33,6 & 97 & 66,4 & $1,40(1,00-1,95)$ & $1,60(1,00-2,55)$ & $0,031^{(*)}$ \\
\hline Graduação & 51 & 23,9 & 162 & 76,1 & & & \\
\hline
\end{tabular}


A equipe de enfermagem apresentou $33,6 \%$ de prevalência para TMC, outros profissionais - 17,9\% e os médicos $-9,1 \%$. A razão de prevalência da equipe de enfermagem foi de 3,69 (IC 95\% 1,69-8,07) comparada aos médicos, conforme observa-se na Tabela 3.

Em relação ao turno de trabalho, a associação de TMC com o período noturno foi estatisticamente significante $(p=0,003)$, os profissionais que trabalhavam à noite apresentaram três vezes mais transtornos mentais que aqueles profissionais que trabalham em mais de um turno (RP: 3,42; IC 95\% 1,41-8,26), sendo que $34,9 \%$ do horário noturno demonstraram tendência a TMC.

Análise de regressão logística revelou que, entre os profissionais de saúde, as mulheres tiveram chance 2,36 vezes mais elevada (IC 95\% 1,09-5,10, $\mathrm{p}=0,028$ ) de apresentar TMC; e entre aqueles com idade inferior a 39 anos houve chance 2,21 vezes mais elevada (IC $95 \% 1,31-3,71, \mathrm{p}=0,003)$ de o rastreamento para TMC ser positivo, mesmo após o ajuste por variáveis identificadas como potenciais confundidoras (categoria profissional, renda e turno de trabalho).

Quanto à idade, encontrou-se maior prevalência de TMC na população jovem, dado que corrobora outros estudos ${ }^{12,26,27}$. Entretando, uma pesquisa, realizada com membros da equipe de enfermagem, evidenciou que pessoas mais velhas apresentam maior frequência de indicativo de $\mathrm{TMC}^{28}$.

A prevalência de TMC foi significativamente maior no sexo feminino, fato este também confirmado por várias pesquisas realizadas em diferentes populações , $10,26-28^{\text {. }}$.

Apesar de a variável renda familiar, após o ajuste como potencial confundidora, não ser estatisticamente significativa, vale ressaltar que quanto menor a renda, maior é a presença de TMC, o que é mencionado por estudo realizado em Pernanbuco ${ }^{6}$. A variável renda pode possibilitar melhores condições de vida, por isso as pessoas com boas condições financeiras apresentam menor prevalência de transtornos mentais, fato este confirmado por outros estudos ${ }^{27,28}$.

Um estudo, realizado em hospitais públicos, demonstrou maior prevalência de TMC em trabalhadores do turno noturno ${ }^{29}$, valores próximos aos encontrados na presente pesquisa. $\mathrm{O}$ trabalho noturno pode trazer prejuízos para a saúde do profissional, sendo considerado algo complexo e que pode estar associado a fatores individuais, às condições de vida e a complexidade da atividade laboral ${ }^{17,18}$.

Apesar de a categoria profissional não ter se mostrado estatisticamente significativa quando ajustadas a outras variáveis, através da regressão logística, faz-se necessário destacar as prevalências em relação à presença de TMC. A análise bivariada mostrou que na equipe de enfermagem a prevalência foi de $33,6 \%$ de TMC, fato este que corrobora outros estudos en- volvendo população similar, nas quais as prevalências variaram de $24,6 \%$ a 33,9\% $\%^{27-30}$. A prevalência de $91 \%$ de TMC entre os médicos foi inferior às encontradas em outros estudos realizados com o mesmo tipo de população, porém, em cenários diferentes, que apresentaram variação entre $17,4 \%$ a $68 \%{ }^{21,22,24,25}$.

\section{Conclusão}

A presença de TMC na população estudada indica que, devido ao caráter subjetivo dos distúrbios não psicóticos, muitos profissionais podem estar subdiagnosticados, e por isso podem não estar se submetendo a um tratamento adequado. Dessa maneira, é necessário propor estratégias, que possam promover a saúde dos trabalhadores, especialmente entre as mulheres, pois foram as que apresentaram maior prevalência de TMC neste estudo. Entretanto, por se tratar de um estudo transversal, em que há uma limitação acerca da causalidade, ressalta-se a necessidade da realização de estudos longitudinais a fim de analisar a influência dos fatores de riscos considerados.

\section{REFERÊNCIAS}

1.Dejours C. A loucura do trabalho: estudo de psicopatologia do trabalho. São Paulo: Cortez- Oberé; 1992. 2.Chaves LD, Ramos LH, Figueiredo EN. Satisfação profissional de enfermeiros do trabalho no Brasil. Acta Paul Enferm. 2011; 24: 507-13.

3.Aiken LH, Sloane DM, Clarke S, Poghosyan L, Cho E, You L, Finlayson M, Kanai-Pak M, Aungsuroch Y. Importance of work environments on hospital outcomes in nine countries. Int J Qual Health Care. 2011; 23: 357-64. 4.Tavares JP, Beck CLC, Magnano TSBS, Greco PBT, Prestes FC, Silva RM et al. Produção cientifica sobre os distúrbios psíquicos menores a partir do self report questionnaie. Rev Enferm UFSM. 2011; 1:113-23

5.Goldberg D, Huxley P. Common mental disorders: a bio-social model. London (UK): Tavistock; 1992.

6.Ludermir AB, Melo Filho DA. Condições de vida e estrutura ocupacional associadas a transtornos mentais comuns. Rev Saude Publica. 2002; 36: 213-21.

7.World Health Organization. The World Health Report 2001. Mental Health: new understanding, new hope. Geneva (Swi): WHO; 2001.

8.Prince M, Patel V, Saxena S, Maj M, Maselko J, Phillips MR, Rahman A. No health without mental health. The lancet. 2007; 370: 859-77.

9.Costa AG, Ludermir AB. Transtornos mentais comuns e apoio social: estudo em comunidade rural da Zona da Mata de Pernambuco, Brasil. Cad Saúde Pública. 2005; 21:73-9.

10.Jansen Karen, Mondin TC, Ores LC, Souza LDM, Konradt CE, Pinheiro RT et al. Transtornos mentais comuns e qualidade de vida em jovens: uma amostra populacional de Pelotas, Rio Grande do Sul, Brasil. Cad Saúde Pública. 2011; 27: 440-8. 
11.Coutinho ESF, Almeida Filho N, Mari JJ. Fatores de risco para morbidade psiquiátrica menor: resultados de um estudo transversal em três áreas urbanas no Brasil. Rev Psiq Clín. 1999; 26: 246-56.

12.Mari JJ, Williams P. A validity study of a psychiatric screening questionnaire (SRQ-20) in primary care in the city of São Paulo. Br J Psychiatry. 1986; 148: 23-6. 13.Goncalves DM, Stein AT, Kapczinski F. Avaliação de desempenho do Self-Reporting Questionnaire como instrumento de rastreamento psiquiátrico: um estudo comparativo com o Structured Clinical Interview for DSM-IV-TR. Cad Saúde Pública. 2008; 24: 380-90.

14.Iacoponi E, Mari JJ. Reability and factor struture of the Portuguese version of Self: reporting questionnaire. Internat J Social Psychiat. 1989; 35: 213-22.

15.Ebling M, Carlotto MS. Burnout syndrome and associated factors among health professionals of a public hospital. Trends Psychiatry Psychother. 2012; 34: 93-100. 16.Mendes SS, Martinho MMF. Trabalho em turnos: estado geral de saúde relacionado ao sono em trabalhadores de enfermagem. Rev esc de enferm USP, 2012; 46:1471-6. 17.Pimenta AM, Kac G, Souza RRC, Ferreira LMBA, Siqueira SMM. Trabalho noturno e risco cardiovascular em funcionários de universidade pública. Rev Assoc Med Bras, 2012; 58:168-77.

18.Silva AA, Rotenberg L, Fischer FM. Jornadas de trabalho na enfermagem: entre necessidades individuais e condições de trabalho. Rev Saude Publica. 2011; 45:1117-26.

19.Silva NS, Esperidião E, Silva KKC, Souza ACS, Calvacante ACG. Perfil profissiográfico de trabalhadores de nível universitário em serviços de saúde mental. Rev enferm UERJ. 2013; 21:185-91.

20.Martins JT, Bobroff MCC,Andrade AN, Menezes GDO. Equipe de enfermagem de emergência: riscos ocupacionais e medidas de autoproteção. Rev enferm UERJ. 2014; 22:334-40

21.Cabana MCFL, Ludermir AB, Silva ÉR, Ferreira MLL, Pinto MER. Transtornos mentais comuns em médicos e seu cotidiano de trabalho. Jornal Bras Psiquiatria. 2007; 56: $33-40$.
22.Carvalho CN, Melo-Filho DA, Carvalho JAG, Amorim ACG. Prevalência e fatores associados aos transtornos mentais comuns em residentes médicos e da área multiprofissional. Jor Bras Psiquiatria. 2013; 62: 38-45. 23.Galvão LLLF, Farias MCS, Azevedo PRM, Vilar MJP, Azevedo, GD. Prevalência de transtornos mentais comuns e avaliação da qualidade de vida no climatério. Rev Assoc Med Bras. São Paulo, 2007; 53:414-20.

24.Braga LC, Carvalho LR, Binder MCP. Condições de trabalho e transtornos mentais comuns em trabalhadores da rede básica de saúde de Botucatu (SP). Ciênc saúde coletiva[online]. 2010; [ citado em 20 jul 2014]. 15: 1585-96. Disponível em: http://www.scielo.br/pdf/csc/ v15s1/070.pdf

25.Barbosa GB, Correia AKS, Oliveira LMM, Santos VC, Ferreira SMS, Martins JDF. Trabalho e saúde mental dos profissionais da estratégia saúde da família em um município do Estado da Bahia, Brasil. Rev Bras Saúde Ocupacional. 2012; 37: 306-15.

26.Gelaye B, Lemma S, Deyassa N, Bahretibeb Y, Tesfaye M, Berhane Y. Prevalence and Correlates of Mental Distress Among Working Adults in Ethiopia. Clin Pract Epidemiol Ment Health, 2012; 8:126-33.

27.Kirchhof ALC, Magnago TSBS, Camponogara S, Griep RH, Tavares JP, Prestes FC, Paes LG. Condições de trabalho e características sócio-demográficas relacionadas à presença de distúrbios psíquicos menores em trabalhadores de enfermagem. Texto contexto - enferm. 2009; 18:215-23.

28.Pinho PS, Araujo TM. Trabalho de enfermagem em uma unidade de emergência hospitalar e transtornos mentais. Rev enferm UERJ. 2007; 15: 329-36.

29.Diniz TB, Silva-Costa A, Griep RH, Rotenberg L. Minor psychiatric disorders among nursing workers - is there an association with current or former night work? Work 41. 2012; 41: 2887-92.

30.Araújo TM, Aquino E, Menezes G, Santos CO, Aguiar L. Aspectos psicossociais do trabalho e distúrbios psíquicos entre trabalhadoras de enfermagem. Rev Saude Publica. 2003; 37: 424-33. 